\title{
Gekürzte Silikon-Drainage als Tourniquet z. B. bei der Operation nach Nesbit
}

Gefäßzügel zur Erzeugung einer artifiziellen Erektion während der Operation sind oft unhandlich. Wir stellen Ihnen eine Alternative vor.

Bei Operationen zur Beseitigung einer Induratio penis plastica oder bei der Versorgung einer Penisfraktur wird in der Regel intraoperativ eine artifizielle Erektion zur Visualisierung der Deviation bzw. einer Le-

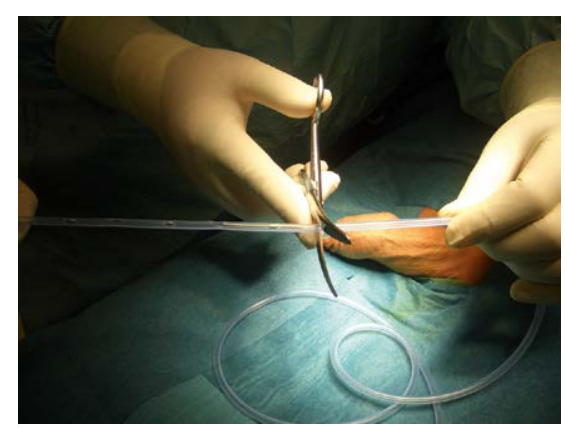

- Abb. 1 Kürzung der Silikondrainage, der wenig zugstabile „Lochteil“ wird entfernt.

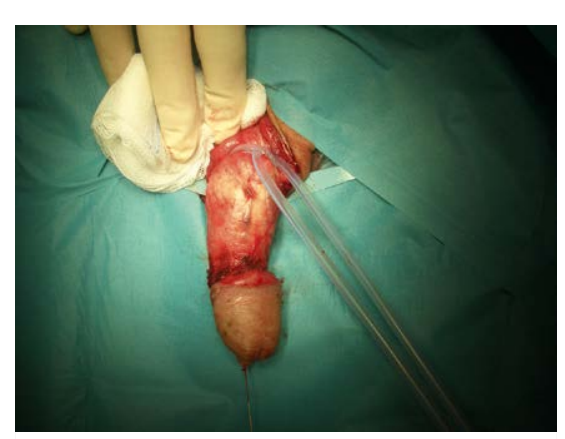

Abb. 2 Umgebaute Drainage als SilikonTourniquet bei der Erzeugung einer artifiziellen Erektion in situ (hier im Rahmen einer OP nach Nesbit). ckage erzeugt. Die hierfür im OP vorhandenen Gefäßzügel sind häufig zu dünn, halten dem notwendigen Zug nicht stand oder „versinken“ im Gewebe, so dass sie schwierig wieder zu lösen sind. Dies wiegt umso schwerer, weil je nach OP-Verlauf der Vorgang mehrfach wiederholt werden muss.

Hier hat sich in unserer Hand die „Zweckentfremdung“ einer 14-Charr-Silikon-Drainage bewährt. Der Lochteil der Drainage wird entfernt - dieser ist nicht zugstabil - und der Rest der immer noch ausreichend langen Drainage um die Penisbasis geschlungen. Es ergibt sich eine effiziente und gewebsschonende Möglichkeit der Erzeugung einer artifiziellen Erektion ( $\triangleright$ Abb. 1,2).

\section{Interessenkonflikt}

Beratungstätigkeit: Dr. Pfleger, Pfizer; Vortragstätigkeit: Allergan, AMS Deutschland, Astellas, Berlin-Chemie, Janssen, Lilly Deutschland, Dr. Pfleger, Pfizer, Pohl-Boskamp; Studienfinanzierung: AMS Deutschland
Autor

Andreas Wiedemann

Korrespondenzadresse

PD Dr. Andreas Wiedemann

Evangelisches Krankenhaus gGmbH

Pferdebachstraße. 27

58455 Witten

awiedemann@diakonie-ruhr.de 